\title{
INSTRUMENTO VÁLIDO PARA AVALIAÇÃO DA QUALIDADE DO CUIDADO NAS ISNTITUIÇÕES DE LONGA PERMANÊNCIA PARA IDOSOS: UMA REVISÃO INTEGRATIVA
}

Márcio de Almeida Alexandre ${ }^{1}$

Nair santos de Souza ${ }^{2}$

\section{RESUMO}

Como a demanda por instituições de longa permanência para idosos (ILPIs) está expandindo, e venha ser relevante para os sistemas de saúde pública discutir o processo de avaliação das condições de saúde dos seus residentes. Em relação a estes acontecimentos, que tem despertado a preocupação a preocupação da sociedade, a interação do idoso em Instituição de Longa Permanência para Idosos (ILPIs) mostra-se como alternativa a ser considerada.

Perante a este cenário, as premissas supracitadas, o presente estudo objetivou identificar, por meio de revisão integrativa, instrumentos de medida construídos especificamente para o gerenciamento e o cuidado de residentes idosos, em instituições de longa permanência.

Em face do objetivo proposto, as demandas assistenciais e gerenciais das ILPI, programa para melhorar a qualidade do cuidado baseada na dimensão Atenção Centrada na Pessoa, utilizando instrumentos como Indicadores Observáveis da Qualidade do Cuidado nas Instituições de Longa Permanência para Idosos (IOQ) adaptado ao contexto nacional na versão brasileira do IOQ.

Torna-se urgente a reformulação de políticas públicas que garantam um sistema de avaliação padronizado dos residentes de ILPIs no Brasil, sendo um desafio fazer com que os instrumentos desenvolvidos consigam se difundir e se efetivar no cotidiano dos profissionais dessas instituições.

Palavras-chave Idoso: Institucionalização, Indicadores de Qualidade, Medidas, Métodos e teorias, Revisão

\footnotetext{
${ }^{1}$ Mestrando em educação pela Logos University international - UniLogos

Professor do colégio Técnico Tableau em Caraguatatuba, Enfermeiro Gestor da ILPI Jardim do Éden em Caraguatatuba-e-mail: aleandrealmar@hotmail.com.//orcid.org/000-0023103-1016
} 
${ }_{2}^{2}$ Mestranda em Educação pela Logos University Intenational - UniLogos

Professora do Centro de Educação Infantil Poiares em Caraguatatuba- prof.Nair@hotmail.com

\section{ABSTRACT}

As the demand for long-term care institutions for the elderly (ILPIs) is expanding, and it will be relevant for public health systems to discuss the process of assessing the health conditions of its residents. In relation to these events, which has aroused the concern of society, the interaction of the elderly in a Long Term Care Facility for the Elderly (ILPIs) is an alternative to be considered.

In view of this scenario, the aforementioned premises, the present study aimed to identify, through an integrative review, measurement instruments built specifically for the management and care of elderly residents, in long-term institutions.

In view of the proposed objective, the care and management demands of the LTCF, a program to improve the quality of care based on the Person-Centered Care dimension, using instruments such as Observable Indicators of Quality of Care in Long-Term Care Institutions for the Elderly (IOQ) adapted to the national context in the Brazilian version of IOQ.

It is urgent to reformulate public policies that guarantee a standardized assessment system for LTCF residents in Brazil, and it is a challenge to ensure that the instruments developed are able to spread and become effective in the daily lives of professionals at these institutions.

Keywords Elderly: Institutionalization, Quality Indicators, Measures, Methods and theories, Review

\section{1- INTRODUÇÃO}

O aumento da população idosa, a demanda por cuidados de longa duração tende a aumentar em todo o mundo, gerando problemas de saúde pública (CREUTZBERG M, 2007, CAMAGOS, MCM. 2014). Consequentemente, surgem as Instituições de Longa Permanência para Idosos (ILPIs) que são organizações complexas, constituídas, majoritariamente, de subsistemas internos, em que a equipe multiprofissional deve se ocupar dos processos de 
gerenciamento e cuidado da saúde global do idoso (CREUTZBERG M, 2007, PIEXAK, DR, 2012)

Por esse ângulo, torna-se função das ILPIs promover a manutenção e a melhoria da qualidade de vida desses idosos, com um monitoramento constante que permita auxiliar no melhor manejo dos residentes (SANTOS,2007) Contudo, FELICIAN et al.2011, declararam a inexistência de instrumentos que avaliem as ILPIs, sob a ótica do cuidado. Para suprir tal deficiência, desenvolveram um estudo preliminar com o propósito de construir um instrumento que identificasse as necessidades reais de uma instituição e de seus residentes. No entanto, existem fortes evidências de que essa temática seja atual, relevante e careça de ser constantemente estudada.

Em relação a estes acontecimentos, que tem despertado a preocupação da sociedade, a internação do idoso em Instituições de Longa Permanência para Idosos (ILPI) mostra-se como alternativa a ser considerada.

Deste modo, embora a saúde do idoso tenha sido anteriormente pesquisada, as discrepâncias e as inadequações na coleta de informações no ato de admissão destes, como residentes, contribuem para uma análise enviesada das condições de saúde, o que pode dificultar a adoção de estratégias eficazes no atendimento dessa população. A falta de homogeneidade de informações dificulta a construção de protocolos que ofereceriam subsídios concretos na tomada de decisões clínicas a curto e longo prazo (RIBEIRO RC, 2009).

Instrumentos de medida são partes integrantes da prática clínica, da avaliação em saúde e de pesquisas, sendo úteis e capazes de apresentar resultados robustos quando desenvolvidos e utilizados cientificamente (COLUCI, 2015). Dessa forma, os métodos e as técnicas de coleta de informações devem garantir indicadores seguros e confiáveis (DE SOUZA, 2013), em que a tomada de decisão vai depender do desenho da pesquisa e dos quanto tais instrumentos são adequados e precisos na avaliação das variáveis de interesse. Portanto, torna-se necessário um mapeamento sistemático e elaborado dos instrumentos de avaliação disponíveis (ALEXANDRE, 2011, PAIXAO,2005).

Os residentes em Instituições de Longa Permanência necessitam de constante monitoramento das condições de saúde, o que é previsto na legislação brasileira (BRASIL 2005, MS, 2006). Sendo assim, entende-se que esses idosos possuem especificidades peculiares que precisam ser consideradas na sua avaliação. Nesse seguimento, instrumentos construídos especificamente para esse público são relevantes para mensurar adequadamente o seu perfil, bem como a realidade vivenciada no contexto institucional. 
No entanto, existe uma lacuna de discussão sobre o processo de avaliação dos residentes nas ILPI's brasileiras e não se encontrou revisões que buscassem traçar um panorama geral sobre os instrumentos de avaliação desenvolvidos especificamente para essa população.

Diante disso, o presente estudo caracteriza-se como uma pesquisa descritiva de revisão integrativa tem a finalidade de " [...] sintetizar resultados obtidos em pesquisa sobre o tema ou questão de maneira ordenada e abrangente. É denominada integrativa porque fornece informações mais amplas sobre um assunto/problema, constituindo, assim, um corpo de conhecimento ( ERGOLE,2014), pressupõe-se que investigações desta natureza possam oferecer o conhecimento dos distintos tipos de ferramentas utilizados em situações de rotina clínica, quer seja no processo de admissão tanto quanto na análise dos efeitos de uma possível ( ALEXANDRE,2011) intervenção sobre os aspectos físico-emocionais, organizacionais ou multidimensionais. Tais resultados e interpretações oferecerão informações a respeito da produção científica sobre o tema, com orientações claras acerca das propriedades destes instrumentos de medida tão heterogêneos, podendo auxiliar profissionais e pesquisadores no seu processo de trabalho.

Perante a este cenário, as premissas supracitadas, o presente estudo objetivou identificar, instrumentos de medida construídos especificamente para o gerenciamento e o cuidado de residentes idosos, em instituições de longa permanência.

\section{MÉTODO}

\subsection{Tipo de Estudo}

Trata-se de um estudo bibliográfico, de natureza qualitativa, do tipo exploratório descritivo, delimitado como revisão integrativa.

Os estudos bibliográficos são realizados a partir do apanhado geral de pesquisas já realizadas, revestidos de importância, por serem capazes de fornecer dados atuais e relevantes relacionados a um determinado tema.

De acordo com WHITTEMORE (2005) a revisão integrativa é um método de pesquisa que possibilita a revisão, crítica e a síntese da literatura representativa acerca de um determinado tema de maneira integrada, permitindo que novas estruturas e perspectivas sobre o tema sejam geradas. Além disso, a revisão integrativa pode ser elaborada com diferentes finalidades, tais como, a definição de conceitos, a revisão de 
teorias ou a análise metodológica dos estudos incluídos de um tópico particular (MENDES; SILVEIRA; GALVÃO, 2008).

Para tanto, seguiu-se as etapas de Revisão Integrativa indicadas por Botelho, CUNHA e MACEDO (2011), conforme consta na figura 1 e descrições nos tópicos seguintes

\section{Fluxograma das Etapas da Revisão Integrativa}

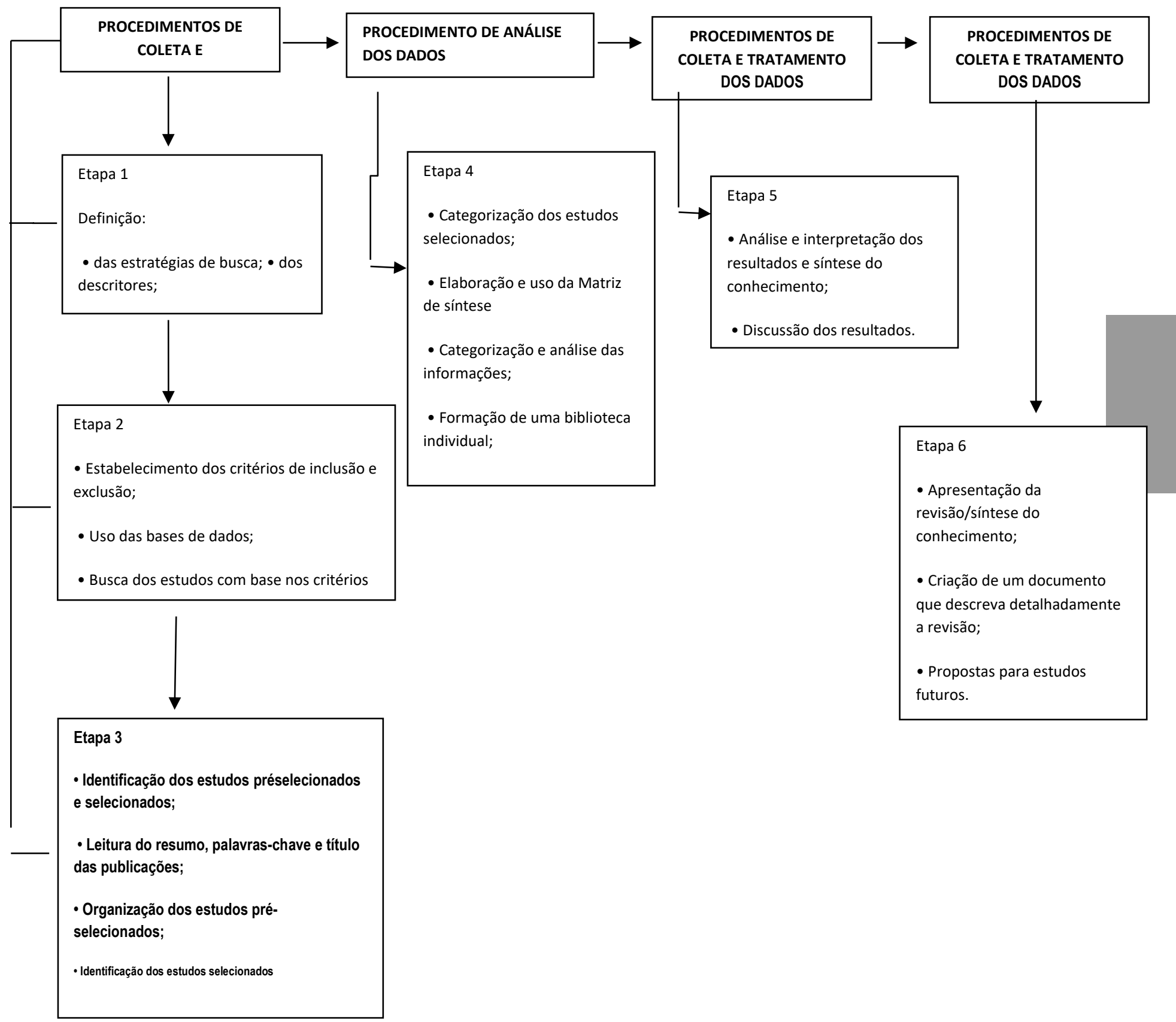

Fonte: Elaborado com base no Manual de Revisão Bibliográfica Sistemática Integrativa (2014). 


\subsection{Procedimentos de Coleta e Tratamento dos Dados}

Etapa 1: Definições das estratégias de busca; dos descritores; e bases de dados.

Uma vez identificados os descritores e abordagem metodológica referente às revisões bibliográficas, a qual permite a inclusão de estudos experimentais e não experimentais para uma compreensão completa do fenômeno ou problema de saúde a ser estudados (SOUZA; SILVA; CARVALHO, 2010), que, no caso do presente estudo, trata-se do Instrumento válido para avaliação da qualidade do cuidado nas instituições de longa permanência para Idoso em bases de dados: BDENF, Portal de Revistas de Enfermagem; SCIELO; LILACS; MEDLINE; INI e CINAHL, por meio de metodologia já existente, utilizando o instrumento adaptado, o instrumento dos indicadores observáveis da qualidade do cuidado nas ILPI (IOQ) foi proposto a partir de um modelo conceitual multidimensional desenvolvido através de uma série de estudos qualitativos, considerando as percepções dos consumidores, prestadores de cuidado e reguladores das ILPI (RANTZ et al., 1999). O IOQ é composto por 30 itens para orientar as observações por diferentes entes sociais agrupados em sete dimensões: (1) Comunicação Interpessoal; (2) Prestação de Cuidados; (3) Gestão da Aparência dos Idosos; (4) Odores; (5) Adequação dos Ambientes Básicos; (6) Acesso aos Ambientes; e (7) Ambiente Familiar. Para cada item ou indicador avaliado, atribui-se pontuação de 1 a 5 , em ordem crescente de qualidade. Assim, o instrumento apresenta uma variação de um mínimo de 30 e máximo de 150 pontos por instituição.

Limita-se a avaliar a qualidade do cuidado nas ILPI a partir de dados referentes à estrutura e processo alusivos à dimensão da Atenção Centrada na Pessoa. Mesmo não havendo uma avaliação das demais dimensões da qualidade em saúde - Efetividade, Eficiência, Segurança, Oportunidade e Equidade-, estudos evidenciam relação positiva da Atenção Centrada na Pessoa com os clássicos resultados de saúde (STEWART, 2001).

Quadro 1 - Critérios de inclusão e de exclusão para a seleção das produções científicas a serem exploradas no estudo

CRITÉRIO

\begin{tabular}{|l|l|l|}
\hline Nacionalidade & $\begin{array}{l}\text { Estudos desenvolvidos no e } \\
\text { sobre o contexto brasileiro }\end{array}$ & $\begin{array}{l}\text { Pesquisas realizadas em } \\
\text { outros países }\end{array}$ \\
\hline Período & $\begin{array}{l}\text { Artigos publicados no período } \\
\text { de } 2000 \text { a } 2020\end{array}$ & $\begin{array}{l}\text { Artigos publicados no } \\
\text { período de 2008 a 2020 }\end{array}$ \\
\hline
\end{tabular}




\begin{tabular}{|l|l|l|}
\hline Tema & $\begin{array}{l}\text { Avaliação da Qualidade do } \\
\text { cuidado em Instituições de } \\
\text { longa permanência para } \\
\text { Idosos. }\end{array}$ & $\begin{array}{l}\text { Avaliação da Qualidade do } \\
\text { cuidado em Instituições } \\
\text { Hospitalares }\end{array}$ \\
\hline $\begin{array}{l}\text { Contexto de } \\
\text { desenvolvimento do estudo }\end{array}$ & $\begin{array}{l}\text { Instituições de longa } \\
\text { permanecia para idosos } \\
\text { públicas ou particulares; }\end{array}$ & $\begin{array}{l}\text { Estudos realizados } \\
\text { exclusivamente em } \\
\text { Instituições Asilares ou } \\
\text { hospitalares. }\end{array}$ \\
\hline Método (Delineamento) & Método (Delineamento) & $\begin{array}{l}\text { Artigos exclusivamente } \\
\text { teóricos }\end{array}$ \\
\hline Participantes & $\begin{array}{l}\text { Gestores (as),Coordenadores } \\
\text { (as) } \\
\text { Enfermeiros(as);Terapeuta } \\
\text { Ocupacionais, Psicólogos; } \\
\text { como principais participantes } \\
\text { da pesquisa. }\end{array}$ & $\begin{array}{l}\text { Estudos realizados } \\
\text { exclusivamente com famílias }\end{array}$ \\
\hline
\end{tabular}

Etapa 3: Identificação dos estudos pré-selecionados e selecionados; Leitura do resumo, palavras-chave e título das publicações; Organização dos estudos préselecionados; e Identificação dos estudos selecionados.

Tendo como base os critérios de inclusão e exclusão elencados para a exploração dos dois periódicos da área da Enfermagem BDENF e Portal das Revista de Enfermagem selecionados, foi identificado um total de 120 artigos publicados, sendo 48 na BDENF e 72 na Portal das Revista de Enfermagem. Prosseguiu-se, então, com a sistematização e seleção da produção científica a ser analisada no presente estudo.

Para esse procedimento, foi necessária a construção de um protocolo de registro de artigos científicos (APÊNDICE A). O instrumento possibilitou a sistematização de todos os estudos encontrados.

A leitura dos títulos, resumos, palavras-chave e introdução dos 120 estudos, culminaram na exclusão de 95 artigos, sendo pré-selecionados 25 estudos para a leitura na íntegra.

À luz dos critérios de inclusão e exclusão, a referida leitura possibilitou a identificação e seleção de 14 estudos para a constituição do corpus de análise do estudo. No quadro 1 é possível ter uma visualização do procedimento realizado. 


\section{FLUXOGRAMA DA BUSCA E SELEÇÃO DOS ESTUDOS}

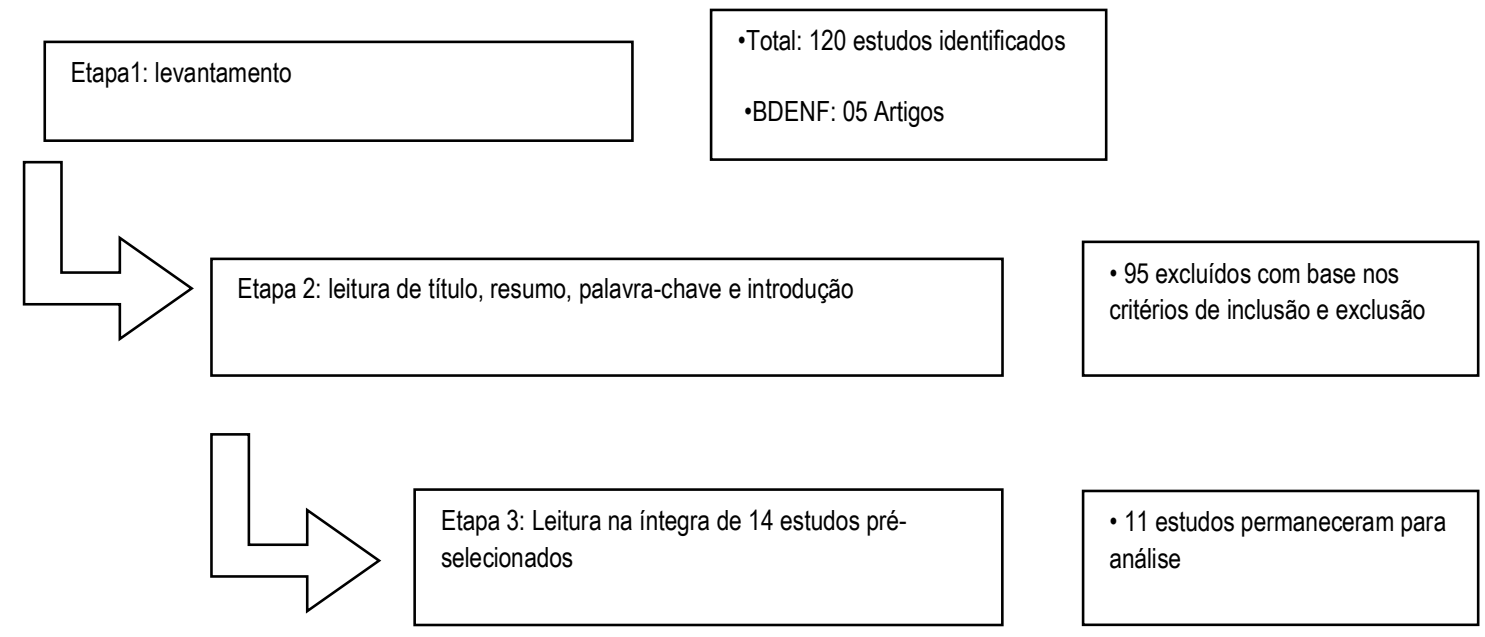

Fonte: Elaboração própria. Nota: Elaborado com base nos dados da BDENF e REV@ENF (2008-2020).

A partir da leitura na íntegra, os artigos foram caracterizados conforme a revista, os(as) autor(as) e ano de publicação, bem como objetivos dos trabalhos. No quadro 2, logo abaixo, estes dados são apresentados.

\section{Quadro 2 - Caracterização dos artigos selecionados}

\section{ARTIGOS \\ OBJETIVO DOS ESTUDOS}

\begin{tabular}{|l|l|}
\hline $\begin{array}{l}\text { OLIVEIRA, } \\
\text { Wagner Ivan }\end{array}$ & $\begin{array}{l}\text { Objetivou-se neste estudo adaptar transculturalmente o IOQ } \\
\text { para avaliar a qualidade do cuidado das ILPIs no contexto } \\
\text { brasileiro. Realizou-se a equivalência conceitual e de itens } \\
\text { para avaliar a pertinência e viabilidade do IOQ à realidade } \\
\text { nacional através do Índice de Validade do Conteúdo (IVC) } \\
\text { junto a um comitê de especialistas formado por 10 } \\
\text { participantes e diretamente envolvidos com o objeto de estudo. }\end{array}$ \\
\hline $\begin{array}{l}\text { BUCHERONI, PM, } \\
2015\end{array}$ & $\begin{array}{l}\text { Descrever e analisar a assistência à saúde do idoso prestada } \\
\text { nas instituições de longa permanência para idosos do } \\
\text { município de São Paulo. }\end{array}$ \\
\hline $\begin{array}{l}\text { OLIVEIRA, } \\
\text { Fagner Ivan }\end{array}$ & $\begin{array}{l}\text { Descrever as etapas iniciais da adaptação transcultural do } \\
\text { Observable Indicators of Nursing Home Care Quality } \\
\text { Instrument (IOQ) usado para avaliar a qualidade do cuidado } \\
\text { nas ILPI. }\end{array}$ \\
\hline $\begin{array}{l}\text { AZEVEDO, Olavo, 2015 } \\
\text { 2011 }\end{array}$ & $\begin{array}{l}\text { Analisar através de uma revisão sistemática de literatura os } \\
\text { indicadores de fragilidade, correlacionado com o ser frágile } \\
\text { etar frágil da pessoa idosa. }\end{array}$ \\
\hline
\end{tabular}




\begin{tabular}{|c|c|}
\hline $\begin{array}{l}\text { PASCOAL, Sérgio } \\
\text { Márcio Pacheco, } \\
2000\end{array}$ & $\begin{array}{l}\text { Delinear procedimentos necessários, para elaboração de } \\
\text { instrumentos de validação de qualidade de vida dos idosos e } \\
\text { definir, a partir de investigação preliminar, os itens que } \\
\text { construirão lista a ser utilizada em etapas futuras para } \\
\text { elaboração efetiva do instrumento }\end{array}$ \\
\hline $\begin{array}{l}\text { FREITAS, Adriana } \\
\text { Valéria da Silva, } \\
2010\end{array}$ & $\begin{array}{l}\text { Discutir o cuidado dispensado ao idoso que reside em } \\
\text { instituição de longa permanência. }\end{array}$ \\
\hline $\begin{array}{l}\text { QUEIROS, } \\
\text { Gleicimara Araujo, } \\
2010\end{array}$ & $\begin{array}{l}\text { Verificar em uma Instituição de Longa Permanência para } \\
\text { Idosos, com características alternativas, a de Qualidade de } \\
\text { Vida percebida pelos sujeitos e seu índice conforme a escala } \\
\text { da OMS. }\end{array}$ \\
\hline $\begin{array}{l}\text { Macêdo de Souza, } \\
\text { I. da S., Teixeira, } \\
\text { K. D., Tavares } \\
\text { Mafra, S. C., \& } \\
\text { Araújo Tinoco, A. } \\
\text { L. (2011) }\end{array}$ & $\begin{array}{l}\text { Avaliar a qualidade de vida (QV) dos idosos residentes na } \\
\text { instituição de longa permanência para idosos de São Vicente } \\
\text { de Paulo de Ubá, MG }\end{array}$ \\
\hline $\begin{array}{l}\text { VITORINO, } \\
\text { Luciano } \\
\text { Magalhães; } \\
\text { PASKULIN, } \\
\text { Lisiane Manganelli } \\
\text { Girardi and } \\
\text { VIANNA, Lucila } \\
\text { Amaral Carneiro, } \\
2012\end{array}$ & $\begin{array}{l}\text { Avaliar a percepção da qualidade de vida (QV) dos idosos de } \\
\text { duas instituições de longa permanência de Pouso Alegre e } \\
\text { Santa Rita do Sapucaí, MG, Brasil }\end{array}$ \\
\hline $\begin{array}{l}\text { Bittar, C. M., } \\
\text { Moscardini, A. F., } \\
\text { Matos Vanzela, I., } \\
\text { Souza, V., \& } \\
\text { Rocha, J. } 2018 .\end{array}$ & $\begin{array}{l}\text { Conhecer a percepção da qualidade de vida de idosos } \\
\text { residentes em instituições de longa permanência para idosos e } \\
\text { sua relação com a espiritualidade. }\end{array}$ \\
\hline $\begin{array}{l}\text { Medeiros, Paulo } \\
\text { Adão,Fortunato, } \\
\text { Artur } \\
\text { Rodrigues,2015 }\end{array}$ & $\begin{array}{l}\text { Identificar instrumentos de medida construídos } \\
\text { especificamente para o gerenciamento e o cuidado de } \\
\text { residentes idosos, em instituições de longa permanência }\end{array}$ \\
\hline $\begin{array}{l}\text { Barbaro, } \\
\text { Alessandra } \\
\text { Marino,2012 }\end{array}$ & $\begin{array}{l}\text { Qualidade de vida e saúde mental de residentes em } \\
\text { Instituições de Longa Permanência para Idosos }\end{array}$ \\
\hline
\end{tabular}




\begin{tabular}{|l|l|}
\hline $\begin{array}{l}\text { Santos, Ariana } \\
\text { Oliveira, Andreotti, } \\
\text { Brenda Couto, 20 }\end{array}$ & $\begin{array}{l}\text { Avaliar a qualidade de vida de idosos residentes em instituição } \\
\text { de longa permanência por meio de uma revisão sistemática }\end{array}$ \\
\hline $\begin{array}{l}\text { Coelho, Pedro } \\
\text { Felipe da Costa } \\
\text { Abreu, Nelsio } \\
\text { Rodrigues ,2018 }\end{array}$ & $\begin{array}{l}\text { Investigar a qualidade de vida subjetiva de idosos que residem } \\
\text { em Instituições de Longa Permanência para Idosos (ILPIs). }\end{array}$ \\
\hline
\end{tabular}

\section{Procedimentos de Análise dos Dados}

Na revisão integrativa, a etapa quatro corresponde ao procedimento de análise de dados representada no quadro 2 .

Figura 2 - Fluxograma etapas de análise

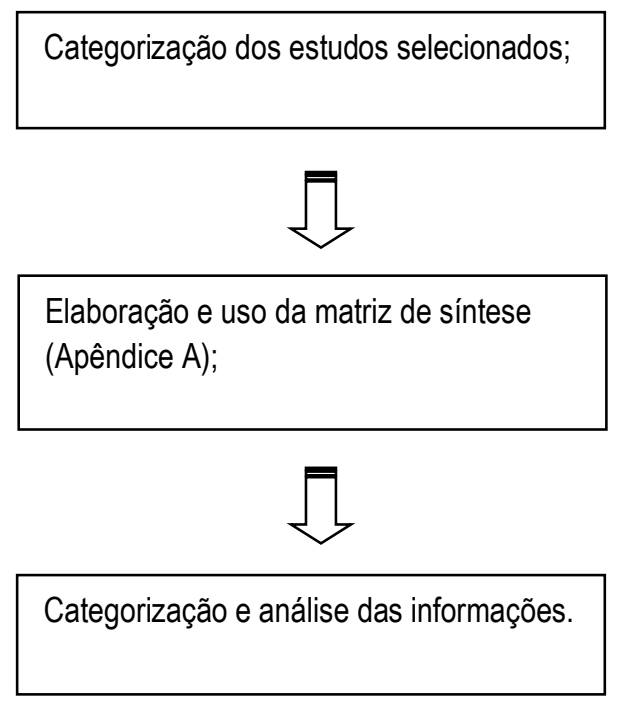

Fonte: Elaboração própria.

No primeiro momento os artigos selecionados foram categorizados no Word, formando categorias conforme os descritores de busca utilizados, descritos na tabela 1. Para análise e discussão dos dados, realizou-se uma síntese de maneira descritiva, apresentando-se dados relevantes e condizentes com as categorias estabelecidas. Conforme as informações foram sendo extraídas dos artigos, estas foram inseridas na matriz de síntese, instrumento que permitiu analisar separadamente cada artigo. A matriz de síntese tem sido utilizada como ferramenta de extração e organização de dados de revisões integrativas 
literatura devido à sua capacidade para resumir aspectos complexos do conhecimento (URSI, 2005; BOTELHO; CUNHA; MACEDO, 2011).

A matriz de síntese objetiva proteger o pesquisador de erros durante a análise. Ela se constitui como marco inicial para auxiliar os investigadores no foco de suas pesquisas. [...] Uma matriz de síntese pode conter informações verbais, conotações, resumos de texto, extratos de notas, memorandos, respostas padronizadas e, em geral, dispor de dados integrados em torno de um ponto ou temas de pesquisa (BOTELHO; CUNHA; MACEDO, 2011, p. 131).

De modo geral, a matriz de síntese é construída com informações sobre aspectos da investigação, e que permita que o pesquisador tenha uma visão geral de dados relacionados a um desempenho de certos pontos. Um dos objetivos da matriz de síntese é proteger pesquisadores de erros durante as análises. Assim, os dados coletados com a matriz servem como ferramenta de interpretação e construção da redação da revisão integrativa. É com essa organização dos dados que sumariza e ordena-se cada pesquisa selecionada, auxiliando o pesquisador no processo de categorização. Ressalta-se que, até o momento, não há um modelo único de matriz de síntese e sua construção depende da interpretação, do domínio do pesquisador acerca do tema em foco e criatividade para organização dos dados (BOTELHO; CUNHA; MACEDO, 2011).

\section{REFERENCIAL TEÓRICO}

Instituição de Longa Permanência de Idosos (ILPI) é definida pela Agência Nacional de Vigilância Sanitária na Resolução da Diretoria Colegiada (RDC) no 283 (ANVISA, 2005) como "instituições governamentais ou não governamentais, de caráter residencial, destinada a domicilio coletivo de pessoas com idade igual ou superior a 60 anos, com ou sem suporte familiar, em condição de liberdade e dignidade e cidadania". (WATANABE; DI GIOVANNI, 2009).

Considera-se ILPI a proposta de uniformização das instituições que prestam assistência aos idosos, garantindo condições de bem-estar físico, emocional e social, em conformidade, entre outros, com o Estatuto do Idoso, com 
a legislação vigente, e com as políticas públicas relacionadas a essa população (CAMARANO \& KANSO, 2010).

A despeito da importância de ILPIs para a qualidade de vida desses indivíduos, a prestação de serviços desses estabelecimentos, em muitos casos, carece de melhorias estruturais e de pessoal qualificado (Oliveira \& Rozendo, 2014). O serviço prestado nas ILPIs é comumente visto como um "ato humanitário", e os idosos não são vistos como consumidores de um serviço pago, mas como meros usuários de uma atividade filantrópica (CAMARANO \& KANSO, 2010).

A maioria das ILPIs, porém, não foi projetada para atender as dificuldades das pessoas idosas; ainda assim, existe uma demanda crescente de idosos a estas instituições, tanto por motivos socioculturais, quanto financeiros. A terceirização do cuidado dos idosos, como solução para atender essa demanda, surgiu com improvisações que nem sempre atendem as suas necessidades, com base na justiça social, nos parâmetros legais, e nas diretrizes arquitetônicas (MILANEZE, 2013 apud VANZIN et al, 2017).

Segundo o Estatuto do Idoso, é garantido aos cidadãos idosos o direito à moradia digna com a família e, caso não seja possível residir com seus familiares, por algum motivo pertinente, é de direito ter acesso às ILPIs públicas ou particulares (LEI n. 10.741, 2003). As ILPIs são espaços mantidos por órgãos governamentais e não governamentais, indicadas a proporcionar assistência integral em caráter residencial em condições de liberdade, destinadas a pessoas acima de 60 anos, com ou sem suporte familiar, de maneira gratuita ou mediante remuneração (AGÊNCIA NACIONAL DE VIGILÂNCIA SANITÁRIA, 2005).

No cenário brasileiro, as ILPIs são comumente confundidas com asilos, que surgiram com o objetivo de fornecer habitação e alimentação aos idosos carentes (CAMARANO \& KANSO, 2010). Todavia, as ILPIs configuram como uma proposta de uniformização das instituições que exercem assistência aos idosos em conformidade com os órgãos relacionados a essa população (MARIN et al., 2012). Tais instituições são obrigadas a fornecer serviços de habitação compatíveis com as necessidades dos idosos (lei n. 10.741, 2003).

As características das práticas de saúde e da sua organização social. Podem ser assim agrupados: relacionados com a disponibilidade e distribuição social dos recursos, (cobertura, acessibilidade e equidade); relacionados com o efeito das ações práticas de saúde implementadas, (eficácia, efetividade); relacionados com o custo de ações (eficiência; relacionados com adequação das ações ao conhecimento técnico cientifico vigente (qualidade técnico-científico); relacionados à percepção dos usuários sobre as práticas (satisfação do usuário). 
A utilização destas noções varia de autor para autor, existindo tentativas de hierarquização das mesmas. A qualidade é vista, com frequência, como aspecto central a ser considerado para avaliação em saúde (DONABEDIAN, 1980; VUORI, 1982). A abrangência destes termos impõe sua definição resulte, frequentemente, de uma composição a partir de outras características do objeto a ser avaliado. Para VUORI (1982), a qualidade dos serviços de saúde seria constituída pela efetividade, eficiência, adequação e qualidade técnica-científica.

DONABEDIAN(1980), que desenvolveu uma discussão sistemática sobre a questão, num dos seus artigos expressava dúvida acerca da natureza da noção de qualidade, ou seja.

[...] Apesar de afirmar ser qualidade uma propriedade do cuidado médico, considerava não estar claro se a mesma era atributo único, uma classe de atributos funcionalmente relacionados ou um conjunto heterogêneo de fenômenos reunidos pelo uso, razões administrativas ou preferências pessoais. Para este autor, a usa de uma definição para a qualidade deveria se iniciar a partir das dimensões do cuidado médico, a saber: a técnico-científico, a relação interpessoal e as "amenidade" (conforto e estética das instalações e equipamentos).

Nesta perspectiva, elaborou inicialmente uma definição, segundo a qual a qualidade poderia ser entendida como a intervenção capaz de alcançar o melhor balanço entre benefício e risco. Foi proposto um modelo unificado para aferição da qualidade envolvendo o balanço risco, benefício e custo do cuidado (DONABEDIAN et al.1982).

[...] Apesar de identificar três possíveis definições para qualidade, a depender do contexto absolutista, individualizado e social, questionava se alguns dos componentes da definição social da qualidade, como o acesso e continuidade, não seriam, na realidade instrumentos para a obtenção da qualidade, e não componentes da mesma, alertando para o risco da perda da utilização analítica do conceito com a sua ampliação. Justificava, assim programas só aproximar-se-ia, segundo 
ele, da aferição da qualidade quanto o mesmo envolvesse a prestação de cuidado individual.

Em artigo posterior (1990) surpreendentemente, passou a considerar a qualidade um fenômeno complexo a ser definido a partir dos seguintes atributos: eficácia, eficiência, efetividade, otimização, aceitabilidade, legitimidade e equidade. Em 1991, definiu as relações interpessoais entre cliente e o profissional, naquilo que estas características têm de "bom" (goodness). Considerava, ainda que a qualidade da dimensão técnica do cuidado devesse ser julgada pela sua efetividade, o que corresponde, em uma última análise, a considerar esses dois termos como equivalentes

A utilidade do IOQ aplica-se para reguladores, profissionais e consumidores dos serviços das ILPI. No âmbito brasileiro, o IOQ se apresenta como um instrumento original e de suma importância para a nova filosofia da Agência Nacional de Vigilância Sanitária de repensar os processos assistenciais através de uma cultura de monitoramento avaliativo (ANVISA, 2011). Ademais, o IOQ fora desenvolvido de forma que mesmo os consumidores com pouco conhecimento sobre as Instituições de Longa Permanência para Idosos possam utilizá-lo para auxiliar as tomadas de decisões para si ou membros da família embasadas na qualidade dos cuidados das ILPI avaliadas. Essa utilidade para os consumidores é importante, pois na ausência de instrumentos de avaliação o preço e a ambiência acabam sendo os únicos parâmetro para julgar a condição de funcionamento das instituições (RANNA, 2012).

Apesar da importância das ILPI como alternativa de cuidados em todo mundo, no Brasil ainda não dispunha de instrumento válido para monitorar a qualidade nesse contexto. Existiam apenas dois instrumentos: (1) fundamentado na Resolução da Diretoria Colegiada (RDC) №. 283/2005, da ANVISA (BRASIL, 2005) e (2) uma ferramenta Avaliação Geriátrica Ampla (AGA), (FREITAS; MIRANDA, 2006). Ambas se limitam por uma considerável especificidade, pois contemplam, principalmente, indicadores de estrutura ou pelo direcionamento exclusivo aos enfermeiros em um contexto complexo e multiprofissional das ILPI.

\section{Instrumentos de avaliação da qualidade do cuidado nas ILPIs}

É pertinente estabelecer padrões de qualidades e rotinas avaliativas da atenção à saúde para os idosos instituicionalizados com o intuito de firmar um processo de avaliação contínua dos serviços prestados, envolvendo mudanças 
organizacionais, culturais e corroborando a solução de problemas que afligem suas atividades diárias.

No Brasil encontramos apenas dois instrumentos de avaliação da qualidade nas ILPIs:

(1) fundamentado na RDC №. 283/2005, da Agência Nacional de Vigilância Sanitária (BRASIL, 2005) e (2) uma ferramenta avaliativa baseada em regulamentações do conselho estadual de enfermagem do Rio Grande do Sul e da própria RDC 283/2005 (FELICIAN, et al., 2011).

Porém, ambas possuem limitações consideráveis e muitas vezes comuns entre elas, já que $73 \%$ das questões da ferramenta avaliativa proposta por FELICIAN et al. (2011), foram fundamentadas na RDC 283/2005. Dentre as limitações, destacam-se: não avaliação da confiabilidade e validade dos instrumentos, ausência de um guia para padronização das observações, grande extensão dos instrumentos e a construção sem considerar a percepção dos principais interessados: usuários. Ademais, a RDC define as condições mínimas de funcionamento das ILPIs e restringe bastante sua avaliação a partir de dados estruturais.

\subsection{Características do instrumento a ser validado}

O instrumento dos indicadores observáveis da qualidade do cuidado nas ILPIs (IOQ) a ser utilizado no estudo, foi proposto a partir de um modelo conceitual multidimensional desenvolvido através de uma série de estudos qualitativos, considerando as percepções dos consumidores, prestadores de cuidado e reguladores das ILPIs (RANTZ et al., 1999).

O IOQ encontra-se na décima versão (RANTZ; ZWYGARTSTAUFFACHER, 2009), composto por 30 itens e um guia para orientar as observações por diferentes entes sociais. Abrange duas escalas gerais de avaliação relacionadas ao processo e estrutura, conforme terminologia adotada por Donabedian (1988), considerando a dimensão da qualidade da atenção centrada na pessoa.

A ferramenta de FELICIAN et al. (2011) apesar de enfatizar também os dados relacionados ao processo, direciona-os de forma exclusiva ao enfermeiro e não a uma equipe multiprofissional. Nos Estados Unidos (RANTZ; ZWYGARTSTAUFFACHER, 2009), Islândia, Canadá (RANTZ et al., 2002) e Coreia do Sul (LEE, 2008) utiliza-se o Observable Indicators of Nursing Home Care Quality Instrument (IOQ) para monitorar a qualidade do cuidado nas ILPIs durante uma 
visita de aproximadamente 30 a 40 minutos. Este instrumento foi desenvoldido a partir do modelo conceitual descrito no tópico anterior desta dissertação após estudos de campo, abrangendo uma pequena amostra internacional e instituições de três estados norte americanos(RANTZ et al., 2006a).

O cuidado em saúde, para ser de qualidade, deve ser individualizado e adequado às necessidades e ao grau de dependência funcional dos idosos, garantindo a atenção às necessidades essenciais (medicamentos, alimentação, higiene pessoal, mudança de decúbito) e prevenindo agravos à saúde. Esses aspectos do cuidado também compuseram uma dimensão da qualidade definida por RANTZ et al.12 (1999).

A qualidade da ILPI na perspectiva dos idosos e trabalhadores responsáveis pelo cuidado é multidimensional e centrada no idoso, inclui aspectos da estrutura e do processo de trabalho. Todas as ações da ILPI devem ter como objetivo a manutenção da autonomia e da independência, a qualidade de vida e o bem-estar dos idosos.

\section{DISCUSSÃO}

Discussões sobre melhoria da qualidade de vida dos idosos institucionalizados em ILPI's foi tratada por ARAUJO, ROCHA, 2016; BALLSTAEDT, 2007; DAWALIBI, et al. 2013; DIAS et al, 2013; FREITAS, SCHEICHER, 2010; MEDEIROS DE ARAUJO NUNES et al, 2010; VITORINO et al, 2013;

O grupo de estudiosos em qualidade de vida da Organização Mundial de Saúde (OMS), The WHOQOL Group, propõe um conceito para qualidade de vida subjetivo, multidimensional e que inclui elementos positivos e negativos: "qualidade de vida é a percepção do indivíduo de sua posição na vida, no contexto da cultura e sistemas de valores nos quais vive, e em relação aos seus objetivos, expectativas, padrões e preocupações". (WHOQOL, 1995, p. 1405). É um conceito amplo e complexo, que engloba a saúde física, o estado psicológico, o nível de independência, as relações sociais, as crenças pessoais e a relação com as características do meio ambiente.

Alguns desses instrumentos foram validados e adaptados ao contexto cultural brasileiro (DINI, QUARESMA e FERREIRA, 2004). Os princípios fundamentais de um envelhecimento com QV descrevem o idoso como proativo, definindo seus objetivos e lutando para alcançá-los, reunindo recursos que são úteis na adaptação à mudança e ativamente envolvido na manutenção do bemestar num mundo mutante e nem sempre propício (FIERRO, 2004). 
O instrumento adaptado (IOQ) limita-se a avaliar a qualidade do cuidado nas ILPIs a partir de dados referentes à estrutura e processo alusivos à dimensão da atenção centrada na pessoa. Mesmo não havendo uma avaliação das demais dimensões da qualidade em saúde: efetividade, eficiência, segurança, oportunidade e acesso, estudos evidenciam relação positiva da atenção centrada na pessoa com os clássicos resultados de saúde (STEWART, 2001). Ademais, segundo Donabedian (1988), os indicadores de processo nos quais se baseia o IOQ é a única forma direta de mensuração da qualidade do cuidado. Além disso, estudos consideram a tríade estrutura, processo e resultados interdependentes, em que boa estrutura facilita bons processos que promovem bons resultados de saúde (COWING et al., 2009).

Além disso, o IOQ fora desenvolvido de forma que mesmo os consumidores com pouco conhecimento sobre as Instituições de Longa Permanência para Idosos possam utilizá-lo para auxiliar as tomadas de decisões para si ou membros da família embasadas na qualidade dos cuidados das ILPIs avaliadas. Essa utilidade para os consumidores é importante, pois na ausência de instrumentos de avaliação o preço e a ambiência acabam sendo os únicos parâmetro para julgar a condição de funcionamento das instituições (RANNA, 2012).

\section{CONSIDERAÇÕES FINAIS}

Espera-se que este artigo, a partir dos seus resultados com base nos aspectos de qualidade empregado nas ILPIs, promova e estimule o bem-estar dos cidadãos brasileiros, explorem as dimensões da qualidade de vida subjetiva das pessoas em ambientes diversos e estimule o desenvolvimento de novas pesquisas para consolidar o sentido destas moradias, em especial, as ILPIs e, a partir dessas investigações, criar e aperfeiçoar novos arranjos espaciais em busca da qualidade de vida.

Revisão integrativa ressaltou que uma equipe multidisciplinar e profissionais especialistas no cuidado ao idoso (enfermeiros ou médicos) podem contribuir para melhorar respostas em saúde dos idosos. Contudo, a utilidade do IOQ aplica-se para reguladores, profissionais e consumidores dos serviços das ILPIs. No âmbito brasileiro, o IOQ se apresenta como um instrumento original e de suma importância para a nova filosofia da Agência Nacional de Vigilância Sanitária de repensar os processos assistenciais através de uma cultura de monitoramento avaliativo.

Os autores discutiram que os estudos do IOQ fora reconhecido como um instrumento válido e confiável no âmbito brasileiro, sendo sugerido seu uso 
para monitorar a qualidade do cuidado nas ILPIs e identificar oportunidades de melhorias. Sua aplicabilidade foi comprovada para diferentes entes sociais, tais como reguladores, potenciais consumidores e profissionais de saúde. O nível de qualidade identificado nas ILPIs participantes foi variável, mostrando pontos positivos nos indicadores de estrutura, relacionados às dimensões de "Odores" e "Utilização dos ambientes básicos", bem como da "Gestão da aparência dos idosos"; além de necessidades de melhorias, prioritariamente, em relação às dimensões "Prestação de cuidados" e "Ambiente familiar". 


\section{REFERÊNCIAS}

1. ARAUJO, F. F.; ROCHA, A. Significados Atribuídos ao Lazer na Terceira Idade: Observação Participante em Encontros Musicais . PODIUM Sport, Leisure and Tourism Review, v. 5, n. 2, p. 38- 55, 2016

2. ALEXANDRE NMC, Coluci MZO. Validade de conteúdo nos processos de construção e adaptação de instrumentos de medidas. Cien Saude Colet 2011; 16(7):3061-3068.

3. ANVISA. Indicador nacional das infecções relacionadas à assistência à saúde. Bol. Inf. Segur. Paciente Qual. Serv. Saúde, v. 1, n. 1, p. 1-12, 2011.

4. BALTHAZAR ML, FIGUEIREDO DBP, CENDES F. Demências. RBM. 2011;68(6):183-193

5. BARKER RO, Craig D, Spiers G, Kunonga P, Hanratty B. Who Should Deliver Primary Care in Long-term Care Facilities to Optimize Resident Outcomes? A Systematic Review. JAMDA 2018; 19:1069e1079

6. BRASIL. Resolução da Diretoria Colegiada - RDC no 283, de 26 de setembro de 2005. Dispõe sobre Regulamento Técnico que define as normas de funcionamento para as instituições de longa permanência para idosos. Agência Nacional de Vigilância Sanitária 2005; 26 set.

7. BRASIL. Ministério da Saúde (MS). Portaria $\mathrm{n}^{\circ} 2.528$ de 19 de outubro de 2006. Aprova a Política Nacional de Saúde da Pessoa Idosa. Diário Oficial da união 2006; 19 out.

8. CAMARGOS MCS. Instituições de longa permanência para idosos: um estudo sobre a necessidade de vagas. Rev. Bras. Estud. Popul 2014; 31(1):211-217.

9. CAMARANO, Ana Amélia; KANSO, Solange. As instituições de longa permanência para idosos no Brasil. Revista brasileira de estudos de população, v. 27 , n. 1 , p. 232-235, 2010.

10. COLUCI MZO, Alexandre NMC, Milani D. Construção de instrumentos de medida na área da saúde. Cien Saude Colet 2015; 20(3):925-936.

11. CREUTZBER M, Goncalves LHT, Sobottka EA, Ojeda BS. A Instituição de Longa Permanência Para Idosos e o Sistema de Saúde. Rev Latino-Am Enfermagem 2007; 15(6):1144-1149. 
12. DESOUSA DA, Moreno AL, Gauer G, Manfro GG, Koller SH. Revisão sistemática de instrumentos para avaliação de ansiedade na população brasileira. Aval. Psicol 2013; 12(3):397-410.

13.DE OLIVEIRA, Wagner Ivan Fonsêca. Validação do Instrumento dos Indicadores Observáveis da Qualidade do Cuidado nas Instituições de Longa Permanência para Idosos Revista Baiana de Saúde Pública, [S.I.], v. 40, n. 4, dez. 2017. ISSN 2318-2660. Disponível em:

<http://rbsp.sesab.ba.gov.br/index.php/rbsp/article/view/2507>. Acesso em: 01 out. 2020 ..

14. DONABEDIAN, A. La calidad de la atención médica: definición y métodos de evaluación. Mexico: La Prensa Médica Mexicana, 1980.

15. DONABEDIAN, A. The seven pillars of quality. Archives of Pathology and Laboratory Medicine, Northfield, v. 114, p. 115-1118, nov., 1990.

16. DONABEDIAN Donabedian, A., 1980a. The definition of quality: A conceptual exploration. In: Explorations in Quality Assessment and Monitoring (A. Donabedian), vol. I, pp. 3-31, Ann Arbor, Michigan: Health Administration Press.

17.ERCOLE FF, Melo LS, Alcoforado CLGC. Revisão integrativa versus sistemática. Rer Min Enferm. 2014;18(1):10.

18. FELICIAN AM, Santos SSC, Pelzer MT, Oliveira AM, Pinho LB. Construção de ferramenta avaliativa direcionada às instituições de longa permanência para idosos: relato de experiência. Rev. Eletr. Enf 2011; 13(3):474-482.

19. FREITAS, E. V.; Miranda, R. D. Parâmetros clínicos do envelhecimento e avaliação geriátrica ampla 2006. In: Freitas, Elizabete Viana de [et. al.]. Tratado de geriatria e gerontologia. Rio de Janeiro: Guanabara-Koogan, p.900-909, 2006.

20. GRASIELA Piuvezam; VIVIANNI Barros Wanderley; RAQUEL Cristina Medeiros de Farias; JOÃO Henrique Câmara da Silveira et al. Análise de indicadores de Qualidade do Cuidado em instituição de Longa Permanência para Idosos (ILPI): Uma revisão Sistemática. In anais do Congresso Internacional de Qualidade em serviços e sistemas de Saúde 2017, . Anais eletrônicos... Campinas, Galoá, 2017. Disponível em:

$<$ https://proceedings.science/qualihosp/papers/analise-de-indicadores-dequalidade-do-cuidado-em-instituicoes-de-longa-permanencia-para-idosos--ilpi--uma-revisao-sist>. Acesso em: 01 out. 2020.

21. LEI $n^{\circ} 10.741$, de 01 de outubro de 2003. Dispõe sobre o Estatuto do Idoso, dá outras providências. Brasília: Diário Oficial da União. 
22. LUCENA, Irapuan Medeiros De et al.. "Instrumento válido para avaliação da qualidade do cuidado nas instituições de longa permanência para idosos: um estudo descritivo". Anais I CNEH... Campina Grande: Realize Editora, 2016. Disponível em: <http://www.editorarealize.com.br/artigo/visualizar/24528>. Acesso em: 01/10/2020 13:39

23. MADDI, S. (2012). Resilience and consumer behavior for higher quality of life. In Mick, D., Pettigrew, S., Pechmann, C., Ozanne, J. (Org.) Transformative Consumer Research for Personal and Collective Well Being: Reviews and Frontiers. New York: Routledge.

24. MILANEZE, G. L. S. (2013). Contribuições para projetos de arquitetura das Instituições de Longa Permanência para Idosos (ILPI), com base na análise de instituições em Criciúma, SC. (225f.). Florianópolis, SC: Dissertação de mestrado em Arquitetura e Urbanismo. Universidade Federal de Santa Catarina

25. PAÍXÃO Júnior CM, Reichenheim ME. Uma revisão sobre instrumentos de avaliação do estado funcional do idoso. Cad Saude Publica 2005; 21(1):7-19.

26.PIEXAK DR, Freitas PH, Backes DS, Moreschi C, Ferreira CLL, Souza MHT. Percepção de profissionais de saúde em relação ao cuidado a pessoas idosas institucionalizadas. Rev. Bras. Geriatr. Gerontol 2012; 15(2):201-208.

27.RANNA, D. S. R. Instituições de longa permanência para idosos na perspectiva do prestador, da legislação e do mercado potencial consumidor. 2012. 97 f. Dissertação (Mestrado em Gerontologia Biomédica) - Pontifícia Universidade Católica do Rio Grande do Sul, Porto Alegre, 2012.

28.RIBEIRO RC, Marin HF. A proposal for a health evaluation tool for the institucionalized aged based on the concept of nursing minimum data set. Rev Bras Enferm 2009; 62(2):204-212.

29.SANTOS SR. Cultura nas instituições de saúde e suas relações com a identidade individual. Cogitare Enferm 2007; 12(2):229-235.

30. STEWART, M. Towards a global definition of patient centred care. BMJ, v. 322, n. 7284, p. 444-5, 2001.

31.VANZIN, Tarcísio; PEREIRA, Marisa Bastos; GONÇALVES, Bibiana Pereira. Observações sistemáticas em Instituições de Longa Permanência para Idosos (ILPI): Considerações arquitetônicas. Revista Kairós: Gerontologia, v. 20, n. 4, p. 195-208, 2017

32.VUORI, H.. A qualidade da saúde. Divulgação em saúde para o debate, Londrina, n. 3, p. 17-24, 1991. 
33.WATANABE, Helena Akemi Wada; DI GIOVANNI, Vera Maria. Instituições de Longa Permanência para idosos (ILPI). BIS. Boletim do Instituto de Saúde (Impresso), n. 47, p. 69-71, 2009.

34.WHOQOL. WORLD HEALTH ORGANIZATION QUALITY OF LIFE GROUP. The World Health Organization Quality of Life assessment (WHOQOL): position paper from the World Health Organization. Social Science and Medicine, v. 41, n. 10 , p. $1403-1409,19$ 


\section{APÊNDICE A- VERSÃO FINAL DO INSTRUMENTO DOS INDICADORES OBSERVÁVEIS DA QUALIDADE DO CUIDADO NAS INSTITUIÇÕES DE LONGA PERMANÊNCIA PARA IDOSOS.}

Versão final da etapa 1 do Observable Indicators of Nursing Home Care Quality Instrument (Português), Rio Grande do Norte, Brasil, 2014.

\section{COMUNICAÇÃO INTERPESSOAL (11 a l6)}

\begin{tabular}{|c|c|c|c|c|c|c|}
\hline I1 & $\begin{array}{l}\text { As conversas entre os residentes e a } \\
\text { equipe de trabalho eram amigáveis? }\end{array}$ & $\begin{array}{c}1 \\
\text { A maioria } \\
\text { não era }\end{array}$ & $\begin{array}{c}2 \\
\text { Poucas eram }\end{array}$ & $\begin{array}{c}3 \\
\text { Algumas } \\
\text { eram }\end{array}$ & $\begin{array}{c}4 \\
\text { Muitas } \\
\text { eram }\end{array}$ & $\begin{array}{c}5 \\
\text { A maioria } \\
\text { era }\end{array}$ \\
\hline 12 & $\begin{array}{l}\text { Quando a equipe de trabalho se } \\
\text { dirigia aos residentes, chamava-os } \\
\text { pelo nome? }\end{array}$ & $\begin{array}{c}1 \\
\text { A maioria } \\
\text { não }\end{array}$ & $\begin{array}{c}2 \\
\text { Poucos sim }\end{array}$ & $\begin{array}{c}3 \\
\text { Alguns sim }\end{array}$ & $\begin{array}{c}4 \\
\text { Muitos sim }\end{array}$ & $\begin{array}{c}5 \\
\text { A maioria } \\
\operatorname{sim}\end{array}$ \\
\hline 13 & $\begin{array}{l}\text { Os residentes e a equipe de trabalho } \\
\text { conheciam } \\
\text { uns aos outros e pareciam confortáveis entre } \\
\text { si (por exemplo, sorriso, contato visual, } \\
\text { toque, etc.)? }\end{array}$ & $\begin{array}{c}1 \\
\text { A maioria } \\
\text { não }\end{array}$ & $\begin{array}{c}2 \\
\text { Poucos sim }\end{array}$ & $\begin{array}{c}3 \\
\text { Alguns sim }\end{array}$ & $\begin{array}{c}4 \\
\text { Muitos sim }\end{array}$ & $\begin{array}{c}5 \\
\text { A maioria } \\
\operatorname{sim}\end{array}$ \\
\hline 14 & $\begin{array}{l}\text { Os residentes e a equipe de trabalho } \\
\text { interagiam uns com os outros de forma } \\
\text { positiva (por exemplo, conversas, humor, } \\
\text { toque, contato visual, } \\
\text { etc.)? }\end{array}$ & $\begin{array}{c}1 \\
\text { A maioria } \\
\text { não }\end{array}$ & $\begin{array}{c}2 \\
\text { Poucos sim }\end{array}$ & $\begin{array}{c}3 \\
\text { Alguns sim }\end{array}$ & $\begin{array}{c}4 \\
\text { Muitos sim }\end{array}$ & $\begin{array}{c}5 \\
\text { A maioria } \\
\text { sim }\end{array}$ \\
\hline 15 & $\begin{array}{l}\text { A equipe de trabalho parecia ser amável } \\
\text { (compassiva, calorosa, gentil)? }\end{array}$ & $\begin{array}{c}1 \\
\text { A maioria } \\
\text { não }\end{array}$ & $\begin{array}{c}2 \\
\text { Poucos sim }\end{array}$ & $\begin{array}{c}3 \\
\text { Alguns sim }\end{array}$ & $\begin{array}{c}4 \\
\text { Muitos sim }\end{array}$ & $\begin{array}{c}5 \\
\text { A maioria } \\
\text { sim }\end{array}$ \\
\hline 16 & $\begin{array}{l}\text { A equipe de trabalho tratava os } \\
\text { residentes com dignidade e respeito? }\end{array}$ & $\begin{array}{c}1 \\
\text { A maioria } \\
\text { não }\end{array}$ & $\begin{array}{c}2 \\
\text { Poucos sim }\end{array}$ & $\begin{array}{c}3 \\
\text { Alguns sim }\end{array}$ & $\begin{array}{c}4 \\
\text { Muitos sim }\end{array}$ & $\begin{array}{c}5 \\
\text { A maioria } \\
\text { sim }\end{array}$ \\
\hline \multicolumn{7}{|c|}{ Total (Some os números circulados e escreva na caixa ao lado) } \\
\hline \multicolumn{7}{|c|}{ PRESTAÇÃO DE CUIDADOS ( 17 a l12) } \\
\hline 17 & $\begin{array}{l}\text { Foram vistos enfermeiros nas áreas comuns } \\
\text { da } \\
\text { instituição? (Observe os crachás ou } \\
\text { pergunte à equipe de trabalho). }\end{array}$ & $\begin{array}{c}1 \\
\text { Não }\end{array}$ & $\begin{array}{c}2 \\
\text { Ocasional- } \\
\text { Mente }\end{array}$ & $\begin{array}{c}3 \\
\text { Algumas } \\
\text { vezes }\end{array}$ & $\begin{array}{c}4 \\
\text { Com } \\
\text { frequência }\end{array}$ & $\begin{array}{c}5 \\
\text { Com } \\
\text { muita } \\
\text { frequênci } \\
\text { a }\end{array}$ \\
\hline 18 & $\begin{array}{l}\text { O(s) enfermeiro(s) pareciam conhecer os } \\
\text { residentes para gerenciar os seus cuidados? } \\
\text { (Pode } \\
\text { ser necessário perguntar à equipe de } \\
\text { trabalho). }\end{array}$ & $\begin{array}{c}1 \\
\text { Não } \\
\text { pareciam }\end{array}$ & $\begin{array}{c}2 \\
\text { Ocasional- } \\
\text { mente }\end{array}$ & $\begin{array}{c}3 \\
\text { Algumas } \\
\text { vezes }\end{array}$ & $\begin{array}{c}4 \\
\text { Com } \\
\text { frequência }\end{array}$ & $\begin{array}{c}5 \\
\text { Com } \\
\text { muita } \\
\text { frequênci } \\
\text { a }\end{array}$ \\
\hline 19 & $\begin{array}{l}\text { A equipe de trabalho ajudava os residentes } \\
\text { com a alimentação? }\end{array}$ & $\begin{array}{l}1 \\
\text { Raramente } \\
\text { visto }\end{array}$ & $\begin{array}{c}2 \\
\text { Ocasional } \\
\text { mente }\end{array}$ & $\begin{array}{c}3 \\
\text { Algumas } \\
\text { vezes }\end{array}$ & $\begin{array}{c}4 \\
\text { Com } \\
\text { frequência }\end{array}$ & $\begin{array}{c}5 \\
\text { Com } \\
\text { muita } \\
\text { frequênci } \\
\text { a }\end{array}$ \\
\hline I10 & $\begin{array}{l}\text { Os residentes eram incentivados a se } \\
\text { deslocarem pela instituição de forma } \\
\text { independente, com ou } \\
\text { sem dispositivo de auxílio, tais como } \\
\text { bengalas, andadores ou cadeiras de } \\
\text { rodas? }\end{array}$ & $\begin{array}{l}1 \\
\text { Raramente } \\
\text { visto }\end{array}$ & $\begin{array}{c}2 \\
\text { Ocasional- } \\
\text { Mente }\end{array}$ & $\begin{array}{c}3 \\
\text { Algumas } \\
\text { vezes }\end{array}$ & $\begin{array}{c}4 \\
\text { Com } \\
\text { frequência }\end{array}$ & $\begin{array}{c}5 \\
\text { Com } \\
\text { muita } \\
\text { frequênci } \\
\text { a }\end{array}$ \\
\hline 111 & $\begin{array}{l}\text { A equipe de trabalho ajudava alguns } \\
\text { residentes a caminhar ou se locomover pela } \\
\text { instituição? }\end{array}$ & $\begin{array}{l}1 \\
\text { Raramente } \\
\text { visto }\end{array}$ & $\begin{array}{c}2 \\
\text { Ocasional- } \\
\text { mente }\end{array}$ & $\begin{array}{c}3 \\
\text { Algumas } \\
\text { vezes }\end{array}$ & $\begin{array}{c}4 \\
\text { Com } \\
\text { frequência }\end{array}$ & $\begin{array}{l}5 \\
\text { Com } \\
\text { muita }\end{array}$ \\
\hline
\end{tabular}




\begin{tabular}{|c|c|c|c|c|c|c|}
\hline & & & & & & $\begin{array}{c}\text { frequênci } \\
a\end{array}$ \\
\hline I12 & $\begin{array}{l}\text { A equipe de trabalho se comunicava com } \\
\text { os residentes confusos de forma positiva } \\
\text { (por } \\
\text { exemplo, falar, tocar sentar-se com eles, } \\
\text { etc.)? }\end{array}$ & $\begin{array}{l}1 \\
\text { Raramente } \\
\text { visto }\end{array}$ & $\begin{array}{l}2 \\
\text { Ocasional- } \\
\text { mente }\end{array}$ & $\begin{array}{l}3 \\
\text { Algumas } \\
\text { vezes }\end{array}$ & $\begin{array}{c}4 \\
\text { Com } \\
\text { frequência }\end{array}$ & $\begin{array}{l}5 \\
\text { Com } \\
\text { muita } \\
\text { frequênci } \\
\text { a }\end{array}$ \\
\hline \multicolumn{7}{|c|}{ Total (Some os números circulados e escreva na caixa ao lado) } \\
\hline \multicolumn{7}{|c|}{ GESTÃO DA APARÊNCIA DOS IDOSOS ( I13 e I14) } \\
\hline I13 & Os residentes estavam vestidos e limpos? & $\begin{array}{c}1 \\
\text { A maioria } \\
\text { não }\end{array}$ & $\begin{array}{l}2 \\
\text { Alguns } \\
\text { estava } \\
\mathrm{m}\end{array}$ & $\begin{array}{l}3 \\
\text { Muitos } \\
\text { estava } \\
m\end{array}$ & $\begin{array}{c}4 \\
\text { A maioria } \\
\text { estava }\end{array}$ & $\begin{array}{l}5 \\
\text { Tod } \\
\text { os } \\
\text { est } \\
\text { ava } \\
\text { m }\end{array}$ \\
\hline I14 & $\begin{array}{l}\text { Os residentes aparentavam estar bem } \\
\text { cuidados (barba feita, cabelo penteados, } \\
\text { unhas limpas e } \\
\text { aparadas)? }\end{array}$ & $\begin{array}{l}1 \\
\text { A maioria } \\
\text { não }\end{array}$ & $\begin{array}{l}2 \\
\text { Alguns } \\
\text { estava } \\
\text { m }\end{array}$ & $\begin{array}{l}3 \\
\text { Muitos } \\
\text { estava } \\
m\end{array}$ & $\begin{array}{l}4 \\
\text { A maioria } \\
\text { estava }\end{array}$ & $\begin{array}{l}5 \\
\text { Tod } \\
\text { os } \\
\text { est } \\
\text { ava } \\
\text { m }\end{array}$ \\
\hline \multicolumn{7}{|c|}{ Total (Some os números circulados e escreva na caixa ao lado) } \\
\hline \multicolumn{7}{|c|}{ ODORES (I15 e I16) } \\
\hline
\end{tabular}

